\title{
Standardization of dynamic magnetic resonance imaging measurements of pelvic organ prolapse: can the PICS line help?
}

\author{
Diaa E. E. Rizk • Ralf Tunn
}

Received: 9 May 2013 / Accepted: 9 May 2013 /Published online: 28 May 2013

(C) The International Urogynecological Association 2013

Dynamic magnetic resonance imaging (MRI) of the pelvis has been rapidly introduced into urogynecological research and practice in recent years mainly to address the shortcomings of clinical assessment systems in women with pelvic organ prolapse (POP). The primary objective was to better select candidates for surgical intervention and choose the appropriate procedure in order to reduce recurrences by allowing accurate identification and objective measurement of prolapse and simultaneous topographic assessment of the pelvis at rest and straining [1-5]. MRI is particularly indicated in women with multi-compartment POP and in those who had undergone previous repair, as imaging can reveal more extensive prolapse than physical examination alone with detection rates similar to other conventional fluoroscopic and ultrasound methods $[1,3$, 4]. Staging of POP using MRI has also been suggested by measuring the perpendicular distance between several reference points and lines in each compartment at rest and after straining [1-5]. The two most commonly used lines are one connecting the inferior aspect of the pubic symphysis to the last coccygeal joint, the pubococcygeal line (PCL), and one extending caudally along the long axis of the symphysis pubis, the midpubic line (MPL). Several MRI staging systems have been published for both of these lines $[1,3,4]$.

Despite the widely accepted role of MRI in supplementing clinical evaluation and management of women with POP, this imaging modality has certain limitations. Most importantly, so

\section{E. E. Rizk}

Department of Obstetrics and Gynecology, Faculty of Medicine, Ain Shams University, Cairo, Egypt

D. E. E. Rizk ( $\square)$

The Canadian Continence Foundation, 671 Elgin Street, Peterborough, Ontario K9H 2E2, Canada

e-mail: rizk.diaa@gmail.com

R. Tunn

Department of Urogynecology, German Pelvic Floor Center, St. Hedwig Hospitals, Berlin, Germany far, there has been no standardized technique for performing MRI examination of the pelvis. Imaging protocols vary according to patient positioning, filling media, pelvic organ opacification, patient maneuvers (i.e., rest, Kegel, Valsalva, evacuation) and MRI sequences and planes [1-5]. Most of the available imaging systems are unable to demonstrate the full extent of POP because patients are examined in a supine position promoting current efforts to image patients in the sitting and upright position using open-magnet MRI units [1, $3-5]$. In fact, sagittal plane images of women with POP that are commonly displayed in the literature as if taken in the upright position had been made in the supine position [6]. A further major problem with MRI studies of the pelvis is the incomplete reproducibility and lack of standardization of the patient effort exerted during straining [3-5].

The anatomical landmarks used for pelvic measurements are easily identified in MRI and this is expected to increase the validity of measurement [5]. The intra- and inter-observer reliability of most MRI measurements is, however, rarely described in POP studies [2]. Furthermore, the reference line used for MRI interpretation of POP is not consistent in all studies as this is often based on radiologist experience and referring physician preference $[1,5]$. In a recent systematic review, seven different reference lines in relation to a wide variety of reference points have been used in different studies with imprecise definition or interchangeable use of some lines, e.g. "MPL/hymenal line" and "PCL/sacrococcygeal inferior pubic point line" [2]. Although the MPL corresponds anatomically to the level of the hymen on cadaveric dissection, the landmark used for clinical staging of POP, the reliability of MRI using the MPL versus clinical findings in women with POP was lower than that of the PCL in most reports $[1,2]$.

It is obvious that we lack a proper validation system for interpreting MRI measurements of POP and we urgently need to reach a consensus on a more standardized and scientifically robust MRI protocol for examination of the pelvis. Betschart et al. review this topic further in a seminal Clinical Opinion 
[6]. The authors discuss the strengths and weaknesses of the current pelvic reference systems for dynamic MRI studies of POP. They clearly demonstrate, after analyzing their database of pelvic MRI scans from 149 women with and without POP, that existing reference lines can produce different measurements because of three factors:

1. The distances measured to the reference line vary with antero-posterior location in oblique line systems.

2. The soft tissue-based reference lines can underestimate organ movement relative to the pelvic bones.

3. The systems defined relative to the scanner are affected by intra- and inter-individual differences in the pelvic inclination angle at rest and strain.

Based on these findings, Betschart et al. propose a standardized MRI measurement system for POP called the pelvic inclination correction system (PICS), which allows standardized measurement of organ displacement in the direction of prolapse by controlling for variation in pelvic inclination, at rest or straining, bony structures, and the body axis [6]. The hypothesis is biologically plausible since variations in pelvic inclination alter the direction of intraabdominal vector forces and the degree of transmission of abdominal pressure onto the pelvic floor, thereby influencing the development of pelvic floor weakness and subsequent prolapse [7].

The PICS line, a new reference line, is a significant novelty in the standardization of pelvic floor measurements using MRI. Classifications and reference systems are widely used in medicine and define groups by similar properties, e.g. the POP-Q system for the clinical staging of POP or the TNM system for the staging of cancer. The PICS line allows the determination of descent of any pelvic organ in a standardized way, independent of the location of the pelvis in the scanner, whether it is tilted, flexed or extended, the size or shape of the pelvis, and the orientation between different examinations or individuals. The advantages of this new system are that it:

1. Is capable of measuring the line of the body axis

2. Results in reliable distance calculation independent of the antero-posterior midsagittal location of the organ because it is not oblique

3. Is related to a bony landmark (pubic bone)

4. Follows the movements of the pelvis

The most significant value, however, is that the PICS line measures the prolapse (or any organ of interest) in the line of gravity that is the natural vector/direction for urogynecological disorders.

The development of the PICS is a product of a fruitful and interdisciplinary collaboration of the Michigan Pelvic Floor Research Group, led by Dr. John DeLancey, which implemented the basic geometrical theorems to solve this measurement problem. Since the group published their first article about the standardized MRI investigation technique in 2001 [8], this contribution 12 years later shows how long the research process takes and how important the ongoing process of standardization of MRI measurements of POP is [6].

Introduction of the new PICS reference line, and hopefully in the future a new reference plane to measure distances out of the midsagittal plane, is an important step in the field of urogynecology toward obtaining standardized and comparable measurements for both clinical practice and research. Until more prospective data are available on the reproducibility of the PICS line in larger and more diverse populations of women with POP, imaging of the pelvic organs using error correction models would assist in establishing a uniform diagnostic and classification system for describing structural defects. It remains to be seen whether the PICS line could also be applied to ultrasound measurements of POP to support routine pre-operative imaging of women with single-compartment prolapse, whether or not surgical treatment is contemplated. We also believe that the PICS line will improve our understanding of the pathogenesis and risks of global pelvic floor dysfunction in women by standardizing the reporting of anatomical variations of the levator ani muscle and endopelvic fascia and correlating this with supportive function.

\section{References}

1. Woodfield CA, Krishnamoorthy S, Hampton BS, Brody JM (2010) Imaging pelvic floor disorders: trend toward comprehensive MRI. Am J Roentgenol 194:1640-1649

2. Broekhuis SR, Fütterer JJ, Barentsz JO, Vierhout ME, Kluivers KB (2009) A systematic review of clinical studies on dynamic magnetic resonance imaging of pelvic organ prolapse: the use of reference lines and anatomical landmarks. Int Urogynecol J 20:721-729

3. Boyadzhyan L, Raman SS, Raz S (2008) Role of static and dynamic MR imaging in surgical pelvic floor dysfunction. Radiographics 28:949967

4. Colaiacomo MC, Masselli G, Polettini E et al (2009) Dynamic MR imaging of the pelvic floor: a pictorial review. Radiographics 29(3):e35

5. Fielding JR (2002) Practical MR imaging of female pelvic floor weakness. Radiographics 22:295-304

6. Betschart C, Chen L, Ashton-Miller JA, DeLancey JOL (2013) On pelvic reference lines and the MR evaluation of genital prolapse: a proposal for standardization using the Pelvic Inclination Correction System (PICS). Int Urogynecol J. doi:10.1007/s00192-013-2100-4

7. Rizk DE, Czechowski J, Ekelund L (2004) Dynamic assessment of pelvic floor and bony pelvis morphologic condition with the use of magnetic resonance imaging in a multi-ethnic, nulliparous, and healthy female population. Am J Obstet Gynecol 191:83-89

8. Tunn R, DeLancey JOL, Quint EE (2001) Visibility of pelvic organ support system structures in magnetic resonance images without an endovaginal coil. Am J Obstet Gynecol 184:1156-1163 